PROTOCOL OPEN

\title{
Predictors for mortality due to acute exacerbation of COPD in primary care: protocol for the derivation of a clinical prediction rule
}

\author{
César Alameda ${ }^{1}$, Ángel Carlos Matía ${ }^{2}$ and Verónica Casado ${ }^{3,4}$ \\ npj Primary Care Respiratory Medicine (2016) 26, 16070; doi:10.1038/npjpcrm.2016.70; published online 20 October 2016
}

\section{BACKGROUND}

Chronic obstructive pulmonary disease (COPD) is the fourth most common cause of death worldwide. ${ }^{1}$ It is expected that this situation will continue to worsen in the coming decades, mainly due to increased tobacco consumption in low- and middle-income countries. ${ }^{2}$ In countries such as Spain, where there has been a decline in smoking, there is already a notable decline in premature mortality and hospitalisations associated with COPD. ${ }^{3,4}$ Throughout the life of an individual living with COPD, frequent exacerbations impair lung function and quality of life, as well as worsen prognosis and raise the associated costs. ${ }^{5-14}$

One area requiring further research in the 'Research Agenda for General Practice / Family Medicine and Primary Health Care in Europe' is the predictive value of history taking and simple clinical examination. ${ }^{15}$ Although some severity score systems have been published, they have been developed in hospital settings and most contain variables that cannot be collected in the context of primary care. ${ }^{16-20}$ We consider it a top priority to define the signs and symptoms that may better predict the severity of an acute exacerbation of COPD (AECOPD), so that we can deliver optimal diagnostic and therapeutic services to our patients.

\section{AIMS}

To derive a clinical prediction rule (CPR) for short-term death following an acute exacerbation of COPD (AECOPD).

\section{METHODS}

Study design

A prospective cohort study in primary care (PC).

Setting

All health centres $(\mathrm{HC})$ of the Spanish provinces of Burgos, Salamanca, Soria, Valladolid and Zamora, totalling 736,183 inhabitants aged between 40 and 79 years. ${ }^{21}$

Inclusion criteria

All persons $\geqslant 40$ years (to exclude patients with potential asthma) attending a $\mathrm{HC}$ of the provinces included in the study between December 2013 and December 2014 who were diagnosed with AECOPD (ICD-9-CM code 491.21) will be included.
Exclusion criteria

Those without a postbronchodilator ratio between forced expiratory volume in one second and forced vital capacity $\left(\mathrm{FEV}_{1} / \mathrm{FVC}\right)<0.7$ in a previous spirometry and those treated for another exacerbation in the past 4 weeks will be excluded. Such cases are classified as 'treatment failure' or 'relapse' and, therefore, are considered a part of the same episode of AECOPD. ${ }^{22}$

Outcome criteria

Details of the outcome criteria are shown in Table 1.

\section{Sample size estimation}

In studies of predictive models, it is customary to include 10 events for each predictor variable in addition to the dependent variable. On the basis of epidemiological studies, the probability of death by AECOPD is $1.2 \%$ in our setting. Therefore, to study 16 possible predictor variables with the prevalence of events set out above, and assuming a 15\% loss, it will be necessary to include 16,292 instances of AECOPD.

\section{Data extraction}

All doctors working in the provinces defined by the study will receive a letter describing the study and requesting their cooperation through an improvement in the collection of clinical data. The data extraction will be carried out through a query to MedoraCyL, the electronic health record of Castile-Leon. The diagnoses made by general practitioners (GPs) will not be reviewed; AECOPD is a clinical diagnosis, and this is a real-world research study. Our objective is to determine the prognosis of what the GPs diagnose as AECOPD with the available resources at the moment of patient care. There will be conditions erroneously diagnosed as AECOPD in the same way that there will be AECOPD erroneously diagnosed as other conditions.

To study the prognosis of the entire AECOPD episode and not the prognosis of each visit to the GP that these patients may perform during the same episode, we will consider visits made 4 weeks after a visit for AECOPD as part of the same episode of AECOPD. ${ }^{22}$ In patients showing multiple values for the same determination during their episode of AECOPD, we will select the one in which the doctor determined that the patient showed the

\footnotetext{
${ }^{1}$ Department of Healthcare Resources, Castile-Leon Regional Health Authority, Valladolid, Spain; ${ }^{2}$ Los Comuneros Health Centre, Burgos Primary Care Authority, Burgos, Spain; ${ }^{3}$ Parquesol Health Centre, West Valladolid Primary Care Authority, Valladolid, Spain and ${ }^{4}$ Department of Medicine, Dermatology and Toxicology, University of Valladolid, Valladolid, Spain.

Correspondence: C Alameda (calamedag@saludcastillayleon.es)

Received 22 April 2016; revised 27 July 2016; accepted 2 August 2016
} 


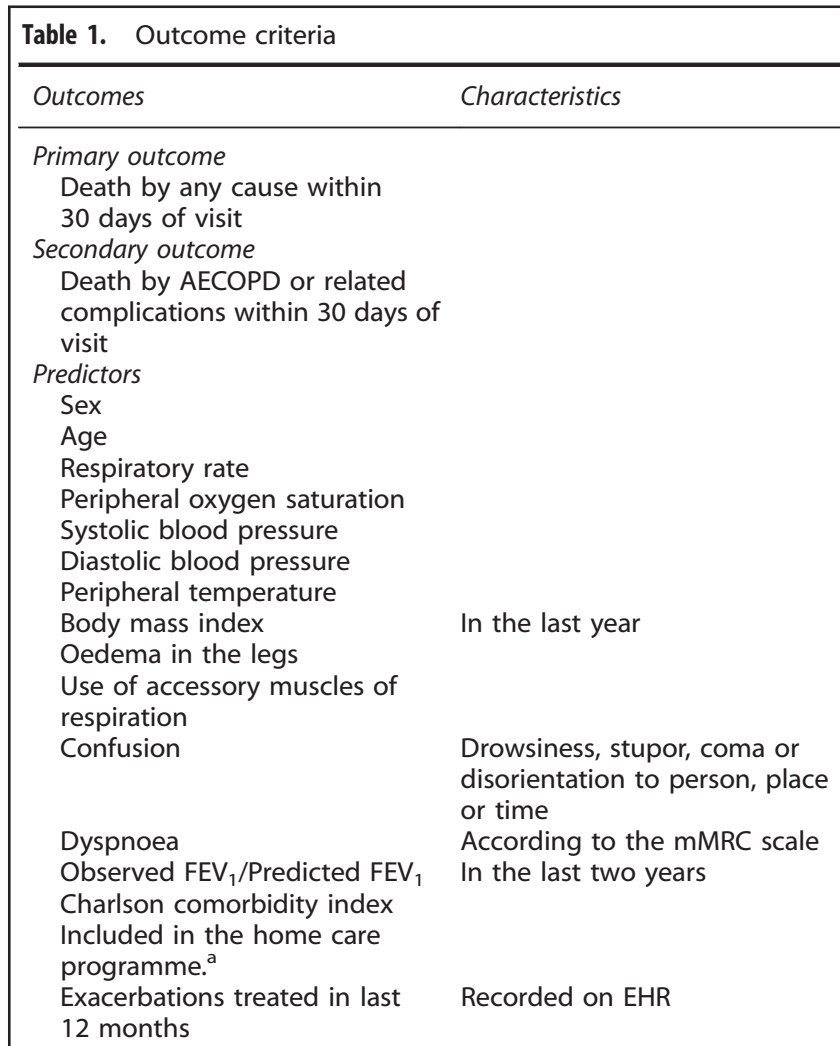

Abbreviations: AECOPD, acute exacerbation of chronic obstructive pulmonary disease; EHR, electronic health record; $\mathrm{FEV}_{1}$, forced expiratory volume in one second; mMRC, modified Medical Research Council.

ancludes people who spend most of their time in bed (who can only leave with the help of others) and those with significant mobility impairments (preventing them from leaving home, except in exceptional cases), regardless of the illness. The expected duration of this disability is more than 2 months.

worst general condition. In patients who visit the $\mathrm{HC}$ several times, death will be determined within 30 days of the last visit.

\section{Methods of data analysis and synthesis}

We will develop a frequency table and a univariate analysis of the predictive ability of each of the predictor variables, both for the primary and for the secondary end points. The statistical significance of the effect of qualitative variables will be studied with the chi-square test. If the expected frequency is $>5$ in more than $20 \%$ of the instances, the Yates correction will be applied. Regarding quantitative variables, normality will be studied with the Kolmogorov-Smirnov test, and statistical significance of the effect will be studied with Student's $t$-test.

We will develop a logistic regression for all-cause mortality at 30 days. We will start from the top model, and variables will be phased out following the method of 'successive steps backward.' This process has been developed taking into account the following features:

- Missing values of a variable will be replaced by the average.

- Non-dichotomous categorical variables will be transformed into dummy variables, which may be combined depending on the frequency distribution.

- Continuous variables will be included in the model after checking their linearity. If not present, the necessary changes will be made.
- We will use the likelihood ratio test to remove predictors from our model. The significance value for this test will be 0.1 .

- The estimation of the regression coefficients will be performed with the maximum likelihood method.

- The goodness of fit will be studied using the log-likelihood function.

After building the model, we will test its fit with the HosmerLemeshow test and determine its accuracy with the area under the ROC (receiver operating characteristic) curve.

We will build the CPR with this model. The CPR will be presented as a score chart on which a score is assigned to each predictor according to its regression coefficient, to facilitate its clinical use. We will determine two cut-off points for the result of the sum of the scores, and three risk categories for AECOPD mortality will be designated as low, medium and high.

These same steps will be repeated to develop a prognostic model for the secondary end point (death by AECOPD or related complications within 30 days of visit). We will compare the prognostic ability between this model and that for all-cause mortality. If no significant differences are found, the model derived for all-cause mortality at 30 days will also be proposed to predict the secondary end point.

The statistical analyses will be performed using IBM SPSS Statistics 21 for Windows (IBM, Chicago, IL, USA).

\section{Ethical approval}

The study protocol has been approved by the local Research Ethics Committees.

\section{SHORT DISCUSSION}

A significant amount is known regarding the predictors of mortality in people with COPD. However, although most people with COPD die from an exacerbation of this condition, there is little published evidence about predictors of mortality by AECOPD, and all such data are based on results from hospital settings. The main strength of this study is that it will be performed in the context of real-world PC and with a large sample size, so that the applicability of the results to clinical practice may be greater than that of other studies. The main limitation of this study is the low rate of the primary end point, which requires a study of only a few predictors to avoid the risk of overfitting; this may make it more difficult to adjust for confounding factors such as comorbidities. Moreover, the predictors of death by AECOPD found in previous studies showed low sensitivity, which could threaten the discrimination of the CPR. The use of real-world data leads to more lost data, especially with regard to spirometry or vital signs that are not systematically measured in PC, such as respiratory rate. The observational design is a limitation itself because it increases the probability of biases. To generalise the findings of this study and to promote its use, validation and clinical impact studies should also be conducted. With this knowledge, clinicians may recognise the signs of poor prognosis in persons with AECOPD and treat them at an earlier stage and avoid diagnostic tests, treatments and hospitalisations in patients who do not require them. The goal of such studies is the optimisation of resources used for the diagnosis and treatment of people with AECOPD.

\section{ACKNOWLEDGEMENTS}

Daniel Araúzo Palacios. General practitioner. Delicias II University Health Centre. Former Director of the Department of Clinic-Assistance Processes and Applications. Castile-Leon Regional Health Authority. The team from the Castile-Leon Comprehensive Assistance for COPD Patient Programme. Pilar Lorenzo Lobato. Department of Information and Communications Technology. Castile-Leon Regional Health Authority. 


\section{COMPETING INTERESTS}

The authors declare no conflict of interest.

\section{FUNDING}

This study is funded by an 'Isabel Fernández' grant from the Spanish Society of Family and Community Medicine (semFYC) and a Biomedicine Research Grant from the Castile-Leon Regional Health Authority.

\section{REFERENCES}

1. GBD 2013 Mortality and Causes of Death Collaborators. Global, regional, and national age-sex specific all-cause and cause-specific mortality for 240 causes of death, 1990-2013: a systematic analysis for the Global Burden of Disease Study 2013. The Lancet 385, 117-171 (2015).

2. Mathers, C. D. \& Loncar, D. Projections of global mortality and burden of disease from 2002 to 2030. PLoS. Med. 3, e442 (2006).

3. Indicadores clave del Sistema Nacional de Salud [Internet]. Madrid: Ministerio de Sanidad, Servicios Sociales e Igualdad; 2014. Available at http://inclasns.msssi.es/ main.html. Accessed on 14 February 2016

4. Encuesta Nacional de Salud 2011-2012 [Internet]. Madrid: Instituto Nacional de Estadística; 2013. Available at http://www.ine.es/jaxi/menu.do?type=pcax is $\&$ path $=/ \mathrm{t} 15 / \mathrm{p} 419 \&$ file $=$ inebase\&L $=0$. Accessed on 14 February 2016.

5. Global Strategy for the Diagnosis, Management and Prevention of COPD. Global Initiative for Chronic Obstructive Lung Disease (GOLD) 2016. Available from: http://goldcopd.org/. Accessed on 14 February 2016.

6. Quintana, J. M. et al. The IRYSS-COPD appropriateness study: objectives, methodology, and description of the prospective cohort. BMC Health Serv. Res. 11, 322 (2011).

7. Toy, E. L., Gallagher, K. F., Stanley, E. L., Swensen, A. R. \& Duh, M. S. The economic impact of exacerbations of chronic obstructive pulmonary disease and exacerbation definition: a review. COPD 7, 214-228 (2010).

8. Doll, H. \& Miravitlles, M. Health-related QOL in acute exacerbations of chronic bronchitis and chronic obstructive pulmonary disease: a review of the literature. Pharmacoeconomics 23, 345-363 (2005).

9. Soler-Cataluña, J. J. et al. Severe acute exacerbations and mortality in patients with chronic obstructive pulmonary disease. Thorax 60, 925-931 (2005).

10. Miravitlles, M. et al. Effect of exacerbations on quality of life in patients with chronic obstructive pulmonary disease: a 2 year follow up study. Thorax $\mathbf{5 9}$ 387-395 (2004).

11. Spencer, S., Calverley, P. M. A., Burge, P. S. \& Jones, P. W. Impact of preventing exacerbations on deterioration of health status in COPD. Eur. Respir. J. 23, 698-702 (2004)

12. Donaldson, G. C., Seemungal, TA. R., Bhowmik, A. \& Wedzicha, J. A. Relationship between exacerbation frequency and lung function decline in chronic obstructive pulmonary disease. Thorax 57, 847-852 (2002).

13. Kanner, R. E., Anthonisen, N. R. \& Connett, J. E. Lower respiratory illnesses promote FEV1 decline in current smokers but not ex-smokers with mild chronic obstructive pulmonary disease: results from the lung health study. Am. J. Respir. Crit. Care Med. 164, 358-364 (2001).

14. Seemungal, T. A., Donaldson, G. C., Paul, E. A., Bestall, J. C., Jeffries, D. J. \& Wedzicha, J. A. Effect of exacerbation on quality of life in patients with chronic obstructive pulmonary disease. Am. J. Respir. Crit. Care Med 157(5 Pt 1): 1418-1422 (1998).

15. Hummers-Pradier, E. et al. Research Agenda for General Practice / Family Medicine and Primary Health Care in Europe (Maastricht: European General Practice Research Network (EGPRN), 2009). Available at http://www.egprn.org/files/user files/file/research_agenda_for_general_practice_family_medicine.pdf. Accessed on 14 February 2016.

16. Quintana, J. M. et al. Predictive score for mortality in patients with COPD exacerbations attending hospital emergency departments. BMC. Med. 12 66 (2014).

17. Steer, J., Gibson, J. \& Bourke, S. C. The DECAF Score: predicting hospital mortality in exacerbations of chronic obstructive pulmonary disease. Thorax 67, 970-976 (2012).

18. Edwards, L. et al. The value of the CRB65 score to predict mortality in exacerbations of COPD requiring hospital admission. Respirology 16, 625-629 (2011).

19. Tabak, Y. P., Sun, X., Johannes, R. S., Gupta, V. \& Shorr, A. F. Mortality and need for mechanical ventilation in acute exacerbations of chronic obstructive pulmonary disease: development and validation of a simple risk score. Arch. Intern. Med. 169, 1595-1602 (2009).

20. Roche, N., Zureik, M., Soussan, D., Neukirch, F. \& Perrotin, D. The Urgence BPCO (COPD Emergency) Scientific Committee and investigators. Predictors of outcomes in COPD exacerbation cases presenting to the emergency department. Eur. Respir. J. 32, 953-961 (2008).

21. Población de Tarjeta Sanitaria de Castilla y León. Diciembre 2012 (Valladolid: Junta de Castilla y León, 2013). Available at http://http://www.saludcastillayleon.es/ institucion/es/sanidadencifras/informes-estadisticos/ordenacion-tematica/ usuarios-sistema-sanitario/poblacion-tarjeta-sanitaria. Accessed on 14 February 2016.

22. Grupo de trabajo de GesEPOC. Guía de práctica clínica para el diagnóstico y tratamiento de pacientes con enfermedad pulmonar obstructiva crónica (EPOC)-Guía española de la EPOC (GesEPOC). Arch. Bronconeumol. 48(Supl 1), 2-58 (2012).

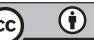

This work is licensed under a Creative Commons Attribution 4.0 International License. The images or other third party material in this article are included in the article's Creative Commons license, unless indicated otherwise in the credit line; if the material is not included under the Creative Commons license, users will need to obtain permission from the license holder to reproduce the material. To view a copy of this license, visit http://creativecommons.org/licenses/ by/4.0/

(c) The Author(s) 2016 